\title{
Anthroposophische Krebstherapie in der Pädiatrie
}

\section{Deutsche Wissenschaftler wollten prüfen, ob die adjuvante, anthropo- sophische Therapie eines kindlichen Medulloblastoms ein Risiko darstellt. Hierzu verglichen sie in einer retro- spektiven Analyse 17 derart behan- delte Fälle mit 34 Fällen, die nur eine konventionelle Therapie erhalten hatten.}

— Die 10-Jahres-Überlebensraten betrugen 58 bzw $57 \%$. Es bestand also kein signifikanter Unterschied bezüglich der
Prognose. Die Autoren schließen daraus, dass die "anthroposophische Therapie, inklusive Mistel, keinen negativen Einfluss auf die Prognose ausübt“.

\section{Kommentar}

In der Vergangenheit wurde wiederholt die Vermutung geäußert, dass Misteltherapie die Wirksamkeit herkömmlicher Krebsmittel beeinträchtigt. Um Klarheit zu schaffen, wurde die hier vorgelegte Analyse durchgeführt. Die Autoren meinen, dass ihre Daten die o.g. Vermutung entkräften. Ich dagegen fürchte, dass dies nicht der Fall ist. Die Untersuchung hat viele Schwächen; u.a. ist die Fallzahl viel zu niedrig, um allgemeingültige Schlüsse zu erlauben. Ferner, so möchte ich meinen, ist eine Krebstherapie vor allem dann brauchbar, wenn sie das Leben verlängert und nicht dann, wenn sie es nicht verkürzt.

\section{E. ERNST -}

\section{Vorsicht mit der Blutdrucksenkung beim Apoplex}

\author{
Es gibt keine Hinweise darauf, dass \\ eine vorsichtige Blutdrucksenkung \\ bei hypertensiven Apoplexie- \\ Patienten zu einer höheren Über- \\ lebensrate oder einem besseren \\ funktionellen Ergebnis führt - eher \\ das Gegenteil.
}

- Bei den meisten Patienten mit Apoplexie ist der Blutdruck entweder bereits chronisch oder akut erhöht. Bei den meisten Ärzten besteht spontan die Neigung, diese erhöhten Blutdruckwerte medikamentös zu senken.

In einer randomisierten, multizentrischen und placebokontrollierten Studie an 146 europäischen Zentren wurden Patienten mit ischämischem oder hämorrhagischem Apoplex und einem systolischen Blutdruck von $140 \mathrm{mmHg}$ oder höher innerhalb von $30 \mathrm{~h}$ nach Beginn der Symptomatik sieben Tage lang entweder mit 4-16 mg Candesartan oder Placebo behandelt. 2029 Patienten konnten eingeschlossen werden, von denen 1017 Candesartan und 1012 Placebo er- hielten. Von 2400 Patienten konnten sechs Monate nach dem Ereignis die Vitalparameter erhoben werden.

Während der siebentägigen Behandlungsperiode lag der Blutdruck unter Candesartan mit 147/82 mmHg signifikant niedriger als unter Placebo mit 152/84 mmHg. Die kombinierten vaskulären Endpunkte, bestehend aus vaskulärem Tod, Myokardinfarkt, erneutem Apoplex und dem funktionellen Ergebnis nach sechs Monaten, waren jedoch in beiden Gruppen nicht signifikant von-einander verschieden. Unter Candesartan kam es zu 120 erneuten vaskulären Ereignissen, unter Placebo dagegen nur zu 111.

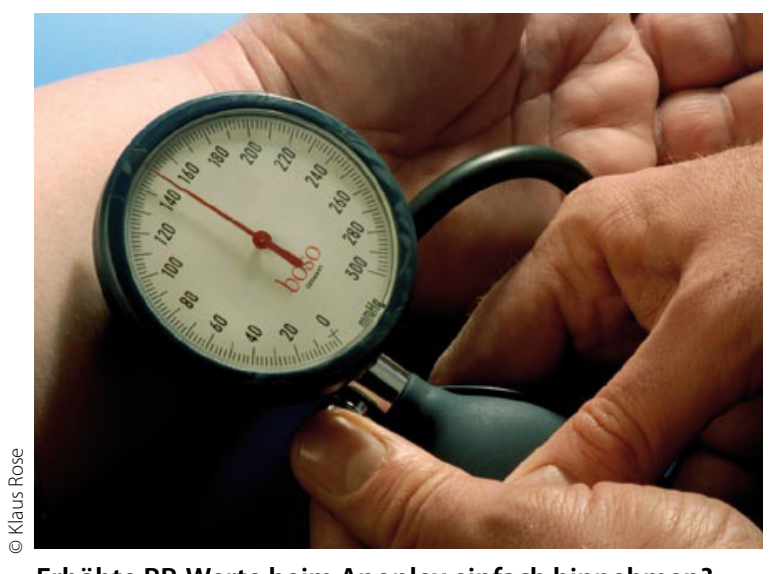

Erhöhte RR-Werte beim Apoplex einfach hinnehmen?
Obwohl dieser Unterschied nicht statistisch signifikant ist, darf doch gefolgert werden, dass die Blutdrucksenkung beim akuten Apoplex zumindest bei mäßigen Blutdruckerhöhungen nicht nur keinen günstigen Effekt hat, sondern sogar eher schadet. In der Followup-Periode entwickelten neun Verumpatienten und fünf Kontrollpatienten eine symptomatische Hypotonie, beim Nierenversagen betrug das Verhältnis 18:13 Patienten.

\section{Kommentar}

So vorteilhaft sich die Blutdrucksenkung, insbesondere mit Angiotensin-Rezeptorblockern zur Prophylaxe von Apoplexien auswirkt, so wenig scheint die Blutdrucksenkung zu nützen, wenn es erst einmal zu einem Apoplex gekommen ist. Es bedarf sicher noch einigen Umdenkens, ehe man erhöhte Blutdruckwerte bei Apoplexie-Patienten in der akuten Phase als solche hinnimmt und von einer akuten Senkung die Finger lässt.

H. S. FüESSL "

- E. C. Sandset et al.

(Korres.: Dr. E. Berge, Oslo University Hospital Ulleval, Departments of Internal Medicine and Cardiology, Kirkeveien 166, NO-0407 Oslo): The angiotensin-receptor blocker candesartan for treatment of acute stroke (SCAT): a randomised, placebo-controlled, double-blind trial. Lancet 377 (2011), 741-750 